\title{
Application of transformers for predicting epilepsy treatment response (Withdrawal Notice)
}

Jiun Choong, Haris Hakeem, Zhibin Chen, Martin Brodie, Nicholas Lawn, et al.

Jiun Choong, Haris Hakeem, Zhibin Chen, Martin Brodie, Nicholas Lawn, Tom Drummond, Patrick Kwan, Zongyuan Ge, "Application of transformers for predicting epilepsy treatment response (Withdrawal Notice)," Proc. SPIE 11884, International Symposium on Artificial Intelligence and Robotics 2021, 1188407 (28 October 2021); doi: 10.1117/12.2601484

Event: International Symposium on Artificial Intelligence and Robotics 2021, 2021, Fukuoka, Japan 


\section{Application of transformers for predicting epilepsy treatment response (withdrawal notice)}

Jiun Choong, Haris Hakeem, Zhibin Chen, Martin Brodie, Nicholas Lawn, Tom Drummond, Patrick Kwan, Zongyuan Ge

Proceedings Volume 11884, International Symposium on Artificial Intelligence and Robotics 20211188407

(2021) https://doi.org/10.1117/12.2601484

Online Publication Date: 28 October 2021

Withdrawn from Publication: 15 November 2021

Publisher's Note: This paper, originally published on 28 October 2021 was withdrawn per author request. 